\title{
DIFERENCIAS DE GÉNERO EN LA ELECCIÓN DE ESTILOS DE VIDA EN ARGENTINA
}

\author{
Rodrigo García Arancibia ${ }^{1}$ \\ Mariano Coronel ${ }^{2}$
}

Recibido: 31/01/2014

Aprobado: 01/04/2014

\begin{abstract}
RESUMEN
Tomando al género como categoría de análisis, en el presente trabajo se estudia la asociación entre los estilos de vida para el caso de Argentina, cuantificando las diferencias reveladas entre varones $y$ mujeres respecto al consumo de alcohol y tabaco, a los hábitos alimenticios y al nivel de actividad física que realizan. Para ello se realiza un análisis multivariado de correspondencia múltiple y se estima un modelo log-lineal de asociación empírica. Los resultados muestran diferencias de género significativas, apoyando la hipótesis de que los hombres afirman su masculinidad y se legitiman como el sexo fuerte por medio de comportamientos poco saludables.
\end{abstract}

PALABRAS CLAVE: ALCOHOL; TABACO; CONSUMO DE FRUTAS Y VERDURAS; ACTIVIDAD FÍSICA; MODELO LOG-LINEAL

\begin{abstract}
Taking gender as a category of analysis, in this paper the association between lifestyles is analyzed for the Argentinean case, quantifying the revealed differences between men and women. Specifically the alcohol and tobacco consumption, dietary habits and level of physical activity are considered in the lifestyle category. A multiple correspondence analysis is performed and a log-linear model of empirical association is estimated. The results show significant gender differences supporting the hypothesis that men affirm their masculinity and are legitimized as the stronger sex through unhealthy behaviors.
\end{abstract}

KEY WORDS: ALCOHOL; TOBACCO; FRUITS AND VEGETABLES CONSUMPTION; PHYSICAL ACTIVITY; LOG-LINEAR MODEL

$1 \quad$ Universidad Nacional del Litoral, Facultad de Ciencias Económicas, Instituto de Economía Aplicada, Código Postal 3000, Argentina.rgarcia@fce.unl.edu.ar

2 Universidad Nacional del Litoral, Facultad de Ciencias Económicas, Instituto de Economía Aplicada, Código Postal 3000, Argentina.mcoronel@fce.unl.edu.ar 


\section{INTRODUCCIÓN}

El marco social e institucional en el que los individuos se desenvuelven juega un rol fundamental en el estado de salud de los mismos. Si bien se observa una mejora en la calidad de vida, la misma no se ha distribuido de manera equitativa en el conjunto de la sociedad; es decir, a medida que el conocimiento acerca de los factores que afectan la salud y las nuevas tecnologías para tratarlos se incrementa, las desigualdades sociales quedan al descubierto (Link, 2008). Esta problemática ha sido abordada desde diferentes ámbitos, tanto académicos como políticos. Entre estos, cabe nombrar aquí, la creación de la Comisión sobre Determinantes Sociales de la Salud en el año 2005 por parte de la Organización Mundial de la Salud (OMS).

Dentro de los determinantes sociales de la salud, el estilo de vida, entendido como un conjunto o patrón de comportamientos, ha demostrado poseer una influencia significativa sobre la calidad de vida de las personas (Balia \& Jones, 2008; Cockerham, 2000; 2004; Contoyanis \& Jones, 2004; Herzfeld, Huffman \& Rizov, 2011; Prus, 2011). De hecho, se observa que el estilo de vida no consiste en una serie de hábitos descoordinados llevados a cabo por individuos sin ninguna relación aparente; sino que, por el contrario, está configurado por rutinas representativas de grupos o clases específicas, siendo el género una variable determinante de esto último (Cockerham, 2005:56-58).

Existe una diversidad de estudios empíricos que constatan diferencias en el estilo de vida practicado por mujeres y hombres (Bryant, Leaver \& Dunn, 2009; Cockerham, Hinote \& Abbott, 2006; Denton, Prus \& Walters, 2004; Denton \& Walters, 1999; Kiefer, Rathmanner \& Kunze, 2005). De la literatura sobre la problemática se desprende que, los hombres ven empeorada su salud a partir de las relaciones de consumo que establecen, mientras que para las mujeres el origen estaría en las relaciones de producción. De acuerdo a Courtenay (2000), ciertos comportamientos riesgosos como el abuso de alcohol y tabaco, están directamente conectados con la identidad masculina, lo que pudiera estar contribuyendo a la menor expectativa de vida de este grupo poblacional.

Teniendo en cuenta la escasez de estudios sobre la relación entre género y estilo de vida para el caso de Argentina, y con el fin de cubrir en parte este vacío, la pregunta que guía la presente investigación es la siguiente: ¿Existen diferencias significativas en las conductas o hábitos llevados a cabo por mujeres y varones en Argentina? Por lo tanto, el objetivo de este trabajo consiste en conocer las diferencias en el estilo de vida practicado por mujeres $y$ hombres en dicho país. Para esto, se trabaja con microdatos provenientes de la Encuesta Nacional de Factores de Riesgo (2005, 2009), y se realiza primeramente un análisis de correspondencia múltiple (MCA); para luego elaborar un modelo log-lineal para computar los cocientes de chances entre pares de variables de interés.

Como hipótesis general, se espera que los varones posean un estilo de vida menos saludable que las mujeres. Dicha conjetura tiene sustento, tanto en el marco teórico desarrollado en la sección segunda del presente trabajo, en el que se adopta una postura de tipo social o cultural, como en los antecedentes empíricos revelados. Específicamente, aunque se observa cierta convergencia en los patrones de consumo de alcohol, comparado con las mujeres en distintas sociedades, el hombre suele incurrir en un mayor abuso de esta sustancia (Wilsnack, R., Wilsnack, S. \& Obot, 2005). De manera similar, la prevalencia en el consumo de tabaco es mayor entre la población masculina, aunque la brecha es más estrecha a medida que se incrementa la emancipación de la mujer (Bauer, Göhlmann \& Sinning, 2006; Hitchman \& Fong, 2011; Lundborg \& Anderson, 2008; WHO, 2007). Respecto a los hábitos alimenticios, se espera que las mujeres mantengan una dieta más balanceada y saludable en términos nutricionales que los varones. Solamente en la realización de actividad física, el hombre presentaría ventajas respecto a la mujer, en términos de una mayor ejercitación física (Frankish, Milligan \& Reid, 1998; Kiefer, Rathmanner \& Kunze, 2005; Rimal, Fletcher \& McWatters, 2000; Zugravu et al., 2009). 
Lo que resta de la presente investigación se estructura de la siguiente manera: en la siguiente sección del trabajo se presenta el enfoque teórico adoptado, describiendo la relación entre género y estilo de vida. Seguidamente se plantea la metodología utilizada, describiendo la fuente de los datos, las variables y modelos a utilizar. La cuarta sección corresponde a la descripción de los resultados y se finaliza con unas breves conclusiones.

\section{GÉNERO Y ESTILO DE VIDA}

El género, como categoría de análisis, surgió en un periodo en el cual tanto activistas como académicos debatían acerca de si los factores que incidían en las diferencias observadas en la salud no reproductiva de mujeres y hombres, eran de tipo biológico, o se sustentaban en convenciones culturales normativas (Krieger, 2003:652).

De manera paulatina, comienza a utilizarse el término "género" para denotar aquellas diferencias que tienen origen en normas culturales, y el "sexo" para marcar las diferencias de tipo biológico. El primero se refiere a un conjunto más amplio de variación acerca de cómo las personas, en diferentes sociedades de todo el mundo, identifican los roles sociales y culturales, valores y comportamientos de hombres y niños, niñas y mujeres (Pollard y Hyat, 1999:2).

Por lo tanto, y considerando que el género es un concepto eminentemente relacional, el interés está orientado hacia las relaciones que establecen hombres y mujeres. Una visión de género, lejos de olvidar a los hombres, los incluye como elemento esencial en las relaciones de poder y desigualdad que afectan a la salud (Borrel, García-Calvente y Martí-Boscà, 2004:3). De acuerdo a Bates, Hankivsky y Springer (2009:1003) equiparar "género y salud" con la salud de las mujeres no permite realizar un correcto entendimiento de la salud de los hombres desde esta perspectiva.

El estilo de vida, desde este enfoque, juega un rol fundamental en la configuración de las relaciones entre hombres y mujeres.

El origen del concepto de estilo de vida puede ser rastreado hasta los teóricos de la sociología clásica, que vieron al mismo como un componente de estratificación. Por ejemplo, como un elemento de la "superestructura" para Marx, o como un elemento que definía a la "clase ociosa" para Veblen, aunque fue Weber el que desarrolló un concepto más acabado, quien marcó su importancia para la evolución y supervivencia del status o clase social (Backet \& Davison, 1995:631). De acuerdo a este último, el estilo de vida opera como punto de encuentro entre las oportunidades (condiciones estructurales) y las elecciones de los individuos, siendo las primeras determinantes o limitantes de las segundas. En un contexto weberiano, las oportunidades están basadas en factores socioeconómicos como la clase o el status, mientras que desde una perspectiva más moderna, existen otras variables de estratificación, como la etnia o el género (Cockerham, 2000:1314).

La mayoría de las actividades que comprenden el estilo de vida tienen lugar fuera de los sistemas de salud, e incluyen un conjunto de comportamientos relacionados con el consumo de alcohol y tabaco, realización de dietas, ejercicio, manejo del estrés, higiene personal, descanso y relajación, uso del cinturón de seguridad, entre otras. Como sostiene Cockerham (2005:5556 ), estos comportamientos son de naturaleza binaria, i.e. saludables o no saludables, lo cual sugiere la evidencia de que para muchas personas, su estilo de vida puede ser caracterizado como generalmente positivo o negativo. García Ruíz (2009:8) define el estilo de vida como un conjunto de conductas características, que tienen un sentido específico para los demás y para el propio sujeto en un lugar y momento concreto. Suele reflejar los valores e ideas básicas de las personas sobre sî mismas y sobre el mundo que les rodea. Por ello, representa un medio para forjar un sentido de sí y para crear símbolos que manifiestan la propia identidad, y precisamente, es la identidad masculina la que está en juego. 
En este sentido, el uso de creencias y comportamientos para definirse como mujer $u$ hombre tienen un profundo impacto en la salud $y$ la longevidad, $y$ es el hombre ante la búsqueda de poder $y$ privilegios, el que frecuentemente daña su propia salud (Courtenay, 2000:1388).

Se construyen modelos o estereotipos acerca de qué es femenino y qué es masculino. Cualidades como la valentía, la dureza, la independencia, forman parte del universo masculino, mientras que la debilidad y la vulnerabilidad tienen una connotación femenina. Más aún, la masculinidad "hegemónica", planteada por Conell y Messerschmidt (2005) no solo supone la contradicción entre lo masculino y lo femenino, sino también entre distintos tipos de masculinidad, estableciéndose diferencias al interior de la población masculina. La masculinidad hegemónica debe ser entendida como un modelo o "tipo ideal" weberiano, utilizado por el hombre para legitimar ideológicamente la subordinación de la mujer, así como también, otros tipos de masculinidades.

A través de la exhibición y la representación de símbolos e ideales constituidos a partir de determinados comportamientos, el hombre refuerza creencias arraigadas en la cultura: es más poderoso $y$ menos vulnerable que la mujer; su cuerpo dispone de mayor eficiencia y resulta superior al femenino; la búsqueda de ayuda y la preocupación por uno mismo son cualidades femeninas; el hombre entre los hombres es aquel para el que la salud y la seguridad son irrelevantes (Courtenay, 2000:1389).

Para Conell y Messerschmidt (2005:844), la dominación del hombre y la subordinación de la mujer constituyen un proceso histórico, no un sistema autosustentable. La dominación masculina es desafiada y requiere de considerable esfuerzo para mantenerla, siendo la elección del estilo de vida un instrumento para tal fin.

Desde este punto de vista, la masculinidad es definida en oposición a comportamientos saludables. Llevar un estilo de vida saludable supondría descender en la escala jerárquica. Por ejemplo, el consumo de alcohol, especialmente en grandes cantidades, ha sido un emblema de la superioridad masculina, $y$ a la vez, permitiría reafirmar poder frente a otros, ya que facilita el comportamiento agresivo (Wilsnack et al., 2005:7-8).

El estilo de vida, entonces, debe ser entendido como medio de construcción de la masculinidad. Desestimando su propia salud y tomando riesgos, a partir del uso de comportamientos poco saludables, el hombre se legitima como el sexo "fuerte". Por ello se espera encontrar hábitos menos saludables en varones que en mujeres en lo referente al consumo de alcohol, tabaco y alimentación; sin embargo respecto a la actividad física, se encuentra que el culto al cuerpo es un ámbito asociado tradicionalmente a lo femenino, pero con la aparición del "nuevo hombre" y la preocupación narcisista por su aspecto, los varones han entrado en la esfera del cuidado del cuerpo a través de la actividad física, la cosmética y la moda (Borrás Catalá, 2007) . Al mismo tiempo, cuando se consideran niveles más exigentes de actividad física, resulta razonable sostener que los varones tienden a realizar una ejercitación más intensa que las mujeres, afianzando con ello su masculinidad más allá de cuestiones biológicas que lo posibiliten.

\section{METODOLOGÍA}

\section{Datos y Variables}

Con el fin de estudiar la asociación entre el género y los estilos de vida, se trabaja con datos secundarios dados por las bases de datos de la Encuesta Nacional de Factores de Riesgo para los periodos 2005 y 2009, elaborada por el Instituto Nacional de Estadísticas y Censos de Argentina (INDEC) y el Ministerio de Salud y Ambiente de la Nación. Las muestras son representativas de una población compuesta por individuos mayores de 18 años (inclusive) que residen en hogares particulares ubicados en zonas urbanas de 5.000 o más habitantes de la República Argentina. En ambas encuestas se presentan diferentes características demográficas y socioeconómicas de los individuos $y$ de sus hogares, $y$ datos de factores de riesgo, tanto en lo que respecta a medidas 
subjetivas (como la auto-percepción de la salud) y medidas objetivas (i.e. peso corporal, colesterol, hipertensión arterial), como a aspectos relacionados a conductas o comportamientos riesgosos: consumo de tabaco y alcohol, hábitos alimenticios y nivel de actividad física. Este trabajo pone el foco en estas últimas variables como indicativas del estilo de vida de los individuos, y considerando el sexo de la persona seleccionada con el propósito de corroborar la existencia de una asociación empírica que apunte a conocer las diferencias de género en relación a diferentes hábitos relacionados a un estilo de vida menos o más saludable.

En relación con el tabaco se considera el hecho de que la persona sea fumadora corriente, dada por la prevalencia de consumo actual de tabaco. De esta manera se define la variable $T$ que es igual a 1 , si el individuo es fumador corriente y cero en caso contrario, considerando como fumadores corrientes a aquellas personas que actualmente fuman todos o algunos días, y que a lo largo de su vida han fumado al menos 100 cigarrillos. La variable $A$ es una variable binaria que indica si la persona abusa habitualmente de bebidas alcohólicas, determinada por la cantidad de tragos de cualquier bebida (ponderada) que toma los días que regularmente consume alcohol. Específicamente, se considera abuso en caso de que la persona haya consumido más de cinco tragos $^{3}$, al menos una vez en los últimos 30 días, de cerveza, vino o bebida fuerte (licores, whisky, vodka, gin o similares) de forma habitual (INDEC, 2005, 2009). Al no diferenciar la cantidad consumida entre varones y mujeres para definir dicha categoría, las diferencias que pudiesen encontrarse en el abuso de alcohol se pueden deber tanto a cuestiones fisiológicas como culturales y sociales que se refieren a la especificidad en términos del género.

Respecto a los hábitos alimenticios se considera el consumo de frutas y verduras; sin embargo, la variable relacionada a dicho consumo no es uniforme en cada encuesta, por lo que las interpretaciones respecto a la misma varían a pesar de que ambas buscan captar el hábito de consumo de frutas y verduras. Para el año 2005, se considera la variable $F V$ que indica si el individuo durante al menos 5 días de la última semana consumió futas y verduras, frutas pero no verduras, verduras pero no frutas o ni fruta ni verdura. Así la variable $F V$ asume tres valores: $F V=1$ indica el no consumo, $F V=2$ indica el consumo de frutas o verduras, ambas opciones excluyentes; y $F V=3$ indica el consumo tanto de frutas como verduras. Para el año 2009 la pregunta relevante busca captar el consumo recomendado de frutas y verduras siguiendo el método STEPS (paso a paso) propuesto por la Organización Mundial de la Salud. En este caso, la variable $F V$ es binaria, siendo igual a 1 cuando el individuo consumió al menos cinco raciones de frutas y verduras en cinco días de una semana típica (semana anterior a la encuesta); e igual a 0 en caso contrario.

Para la actividad física se consideran tres niveles definidos en la encuesta. Específicamente se tiene una variable categórica $A F$ tal que, $A F$ es igual a 3 si el individuo realiza un nivel intenso de actividad física, el que corresponde a por lo menos 3 días de actividad física acumulando 1500 METS $^{4}$-minuto por semana o bien 7 o más días de cualquier combinación de caminata, moderada o intensa, acumulando un mínimo de 3.000 METS-minuto por semana. $A F$ es igual a 2 si la actividad física es moderada, lo cual equivale a 3 o más días de actividad intensa durante al menos 20 minutos por día, o al menos 5 días de ejercicio moderado o caminata al menos 30 minutos por día; o 5 o más días de cualquier combinación de caminata, moderada o intensa, acumulando un mínimo de 600 METS-minuto por semana. Por último, cuando $A F$ corresponde a1, indica un nivel bajo de actividad física, definido como aquel nivel que no llega a ser moderado ni mucho menos intenso.

3 Entendiendo como 'trago' una lata de cerveza, una copa de vino y una medida de bebida fuerte (INDECENPreCoSP, 2008) 
La distribución muestral de las categorías analizadas según género ${ }^{5}$ se presenta en el cuadro 1. En la categoría de consumo de alcohol se observan importantes diferencias en la proporción de varones y mujeres que abusan de bebidas alcohólicas, mostrando un incremento aproximado del $100 \%$ entre 2005 y 2009 tanto para varones como para mujeres. Por el contrario, en el consumo de tabaco se observa una leve reducción de la participación en el consumo corriente, lo que puede deberse a políticas públicas dirigidas con tales fines, como la prohibición de fumar en lugares públicos y cerrados, $y$ la mayor publicidad negativa expuesta en las etiquetas $y$ en diferentes medios gráficos $y$ visuales. A pesar de esta menor propensión al consumo para ambos sexos, se revela una mayor proporción de varones fumadores que de mujeres en los dos años analizados.

Para el caso del consumo de frutas y verduras, se observa que para la muestra de 2005 las mayores diferencias entre varones y mujeres se dan en la proporción que participa consumiendo tanto frutas como verduras en al menos 5 días de una semana típica. Para 2009 se observa como la mayor exigencia que presupone la categoría $F V$, en este caso, implica una baja participación del consumo recomendado de frutas y verduras, además no existen diferencias entre sexos en las proporciones muestrales.

Para la actividad física se observa una mayor proporción de mujeres que realizan actividad física moderada. Por el contrario, el porcentaje de hombres que realiza actividad física intensa llega a duplicar al de mujeres. Al comparar 2009 con 2005 se observa una menor proporción de individuos que realizan ejercitación moderada, incrementándose tanto el porcentaje de individuos más sedentarios como de aquellos que realizan actividad intensa.

\section{CUADRO 1 \\ PROPORCIONES SEGÚN GÉNERO. 2005-2009. EN PORCENTAJE}

\begin{tabular}{|c|c|c|c|c|c|c|c|}
\hline & & \multicolumn{3}{|c|}{2005} & \multicolumn{3}{|c|}{2009} \\
\hline & & Mujer & Varón & Total & Mujer & Varón & Total \\
\hline \multirow{2}{*}{$\begin{array}{c}\text { Alcohol } \\
(A)\end{array}$} & No abuso & 98 & 87 & 93 & 96 & 77 & 88 \\
\hline & Abuso & 2 & 13 & 7 & 4 & 23 & 12 \\
\hline \multirow{2}{*}{$\begin{array}{c}\text { Tabaco } \\
(T)\end{array}$} & No fumador & 75 & 63 & 69 & 78 & 67 & 73 \\
\hline & Fumador corriente & 25 & 37 & 31 & 22 & 33 & 27 \\
\hline \multirow{3}{*}{ Frutas y Verduras $(F V)$} & No consume & 28 & 38 & 32 & 95 & 95 & 95 \\
\hline & Fruta o verdura & 37 & 37 & 37 & \multirow{2}{*}{5} & \multirow{2}{*}{5} & \multirow{2}{*}{5} \\
\hline & Fruta y verdura & 35 & 25 & 31 & & & \\
\hline \multirow{3}{*}{ Actividad Física $(A F)$} & Bajo & 47 & 42 & 45 & 59 & 52 & 57 \\
\hline & Moderado & 46 & 42 & 44 & 31 & 30 & 30 \\
\hline & Intenso & 7 & 16 & 11 & 10 & 18 & 13 \\
\hline Observaciones & & 23.565 & 17.827 & 41.392 & 19.704 & 15.028 & 34.732 \\
\hline
\end{tabular}

Fuente: Elaboración propia con base en ENFR 2005-2009.

$5 \quad$ A partir de aquí, la variable sexo será tomada como proxy del 'género'. Si bien la dimensión del género es más comprensiva que la del sexo, al entender la subdivisión de varones y mujeres cómo categorías de género se está presuponiendo que las diferencias que se analizan en los estilos de vida comprenden motivaciones socio-culturales que van más allá de la cuestión biológica. A pesar de las limitaciones de tomar dicha proxy, esta postura reduccionista permite trabajar empíricamente con la disponibilidad de variables que en general presentan las encuestas no focalizadas en la cuestión de género. 


\section{Análisis de asociación empírica}

Para estudiar la posible existencia de asociación entre estas cuatro variables, en primer lugar se realiza un análisis de correspondencia múltiple (MCA). La metodología MCA es una técnica del Análisis Multivariado, la cual consiste en estudiar la distancia entre un par o varias variables categóricas, brindando un instrumental gráfico bidimensional de gran utilidad y de fácil interpretación para el análisis de asociaciones (Ozden \& Mendes, 2005; Aktürk, Gün \& Kamuk. 2007).

Adicionalmente se especifica un modelo log-lineal, en donde se estiman los cocientes de chances entre pares de variables, cuantificando con ello las diferencias reveladas entre hombres $y$ mujeres respecto a las distintas categorías relacionadas a los estilos de vida.

Como variable respuesta del modelo, se toma la variable aleatoria $Y_{i j k l m}$ que representa el número de personas con las características $i, j, k, l$ y $m$ determinadas por el género $(G)$, el consumo de tabaco $(T)$, el abuso de bebidas alcohólicas $(A)$, el consumo de frutas y verduras $(F V)$ y el nivel de actividad física $(A F)$.

Bajo el supuesto de que $Y_{i j k l m} \sim P\left(\mu_{i j k l m}\right)$ i.e. Poisson de parámetro $\mu_{i j k l m}$, luego en términos generales el modelo puede escribirse como:

$$
\log \left(\mu_{i j k l m}\right)=\gamma+\gamma_{i}^{G}+\gamma_{j}^{T}+\gamma_{k}^{A}+\gamma_{l}^{F V}+\gamma_{m}^{A F}+\sum_{g=2}^{5} I^{g}(G, T, A, F V, A F)
$$

Donde $\mu_{i j k l m}$ es la cantidad promedio de casos con las características $i, j, k, l$ y $m$. Con $I^{g}(G, T, A, F V, A F)$ se representan las interacciones de orden $g$ de las cinco variables, i.e. $g=2$ se tienen todas las interacciones dobles entre todos los pares de las variables. La interacción de mayor orden $(g=5)$ viene dada por $I^{5}(G, T, A, F V, A F)=\gamma_{i}^{G} \times \gamma_{j}^{T} \times \gamma_{k}^{A} \times \gamma_{l}^{F V} \times \gamma_{m}^{A F}$ y su incorporación especificaría un modelo saturado. En general, son preferibles los modelos no saturados debido a que el ajuste suaviza los datos muestrales y permite interpretaciones más simples del modelo (Agresti, 2002: 316). Por lo tanto se busca un modelo que presente un buen ajuste y sea parsimonioso (menor $g$ ).

En caso de que $I^{g}(G, T, A, F V, A F) \equiv 0$ para todo $g$, se tiene un modelo de independencia, y para $I^{p}(G, T, A, F V, A F) \equiv 0$ con $p \geq 3$ y $I^{2}(G, T, A, F V, A F) \neq 0$ se tiene un modelo de asociación homogénea.

El modelo (1) es estimado por máxima verosimilitud. La utilización de modelos log-lineales no solo permite conocer el grado de asociación entre un conjunto de variables categóricas, sino que también con los resultados de su estimación pueden computarse los denominados cocientes de chances (odds ratios, $O R$ ) entre pares de variables, dando una medida cuantitativa específica sobre las asociaciones estudiadas. Cuando se tiene un modelo con solo interacciones dobles, la interpretación de los odds ratios es mucho más sencilla que cuando el modelo incluye interacciones de orden superior. En este último caso, los cocientes de chances 'correctos' entre un par de variables son los condicionados (Agresti, 2002).

Por los objetivos perseguidos en el presente trabajo, los cocientes de chance de interés serán aquellos computados para los pares que involucran al género, es decir: $\{(G, T) ;(G, A) ;(G, F V) ;(G, A F)\}$. En particular, los cocientes de chances se calculan a partir de los predichos, que resulta equivalente a su cálculo a partir de los coeficientes $\gamma_{x}^{Z}$. Para el par $(G$, $T)$ con $G=(1.0)$ (varon, mujer) y $G=(1,0)=$ (fumador corriente, no fumador), los vendrán dados por

$$
\left.O R_{G, T}\right|_{A=k, F V=l, A F=m}=\left.\frac{\widehat{\mu}_{G=1, T=1} \times \widehat{\mu}_{G=0, T=0}}{\widehat{\mu}_{G=1, T=0} \times \widehat{\mu}_{G=0, T=1}}\right|_{A=k, F V=l, A F=m}
$$


Donde $A=$ (abuso habitual de alcohol, no abusa); = (1,0); $F V=$ (frutas y verduras, frutas o verduras, no consumo $)=(1,2,3)$ para el año 2005 y $F V=$ (consumo recomendado de frutas $y$ verduras, no Consumo o consumo menor al recomendado) $=(1,0)$ para el año 2009; y $A F=$ (bajo, moderado, intenso $)=(1,2,3)$.

Para el par $(G, A)$ los $O R$ condicionados serán

$$
\left.O R_{G, A}\right|_{T=j, F V=l, A F=m}=\left.\frac{\widehat{\mu}_{G=1, A=1} \times \widehat{\mu}_{G=0, A=0}}{\widehat{\mu}_{G=1, A=0} \times \widehat{\mu}_{G=0, A=1}}\right|_{T=j, F V=l, A F=m}
$$

Para el par los condicionados para el año 2005, tomando como base el no consumo de frutas $y$ verduras, vendrán dados por

$$
\begin{aligned}
& \left.O R_{G, F V(2,1)}\right|_{T=j, A=k, A F=m}=\left.\frac{\widehat{\mu}_{G=1, F V=2} \times \widehat{\mu}_{G=0, F V=1}}{\widehat{\mu}_{G=1, F V=1} \times \widehat{\mu}_{G=0, F V=2}}\right|_{T=j, A=k, A F=m} \\
& \left.O R_{G, F V(3,1)}\right|_{T=j, A=k, A F=m}=\left.\frac{\widehat{\mu}_{G=1, F V=3} \times \widehat{\mu}_{G=0, F V=1}}{\widehat{\mu}_{G=1, F V=1} \times \widehat{\mu}_{G=0, F V=3}}\right|_{T=j, A=k, A F=m}
\end{aligned}
$$

Para el año 2009 el $O R$ del par $(G, F V)$ es similar pero para dos niveles $F V=0,1$.

Por último, para la actividad física se estiman también los $O R$ condicionados en relación al género, tomando como base el nivel bajo de actividad física $(A F=1)$; es decir:

$$
\begin{aligned}
\left.O R_{G, A F(2,1)}\right|_{T=j, A=k, F V=l} & =\left.\frac{\widehat{\mu}_{G=1, A F=2} \times \widehat{\mu}_{G=0, A F=1}}{\widehat{\mu}_{G=1 A, F=1} \times \widehat{\mu}_{G=0, A F=2}}\right|_{T=j, A=k, F V=l} \\
\left.O R_{G, A F(3,1)}\right|_{T=j, A=k, F V=l} & =\left.\frac{\widehat{\mu}_{G=1, A F=3} \times \widehat{\mu}_{G=0, A F=1}}{\widehat{\mu}_{G=1, A F=1} \times \widehat{\mu}_{G=0, A F=3}}\right|_{T=j, A=k, F V=l}
\end{aligned}
$$

Para cada $O R$ se contrasta la hipótesis $O R=1$ a los efectos de conocer si las diferencias de género empíricamente detectadas, son estadísticamente significativas. Para elaborar un tipo t-test se procede a trabajar con la distribución muestral del logaritmo $\ln (O R)$, la que es aproximadamente normal con error estándar igual a $E E_{\ln O R}=\sqrt{\frac{1}{\widehat{\mu}_{11}}+\frac{1}{\widehat{\mu}_{01}}+\frac{1}{\widehat{\mu}_{10}}+\frac{1}{\widehat{\mu}_{00}}}$ donde los $\mu_{i j}$ representan los conteos predichos utilizados en el cómputo del $O R$ específico. Con esto se obtiene un intervalo de confianza $\exp \left[\ln (O R) \pm z_{\alpha} E E_{\ln O R}\right]$ para el $O R$ original, lo que permite contrastar la homogeneidad en cada caso.

\section{RESULTADOS}

En el anexo se presentan los resultados de la comparación de diferentes modelos loglineales anidados, $y$ los parámetros estimados para aquellos modelos seleccionados en función de su ajuste para los años 2005 y 2009 (cuadros A2 y A3). En el cuadro A1 se tiene el valor del estadístico $G^{2}$ con sus correspondientes grados de libertad, para diferentes modelos anidados, calculado para realizar contrastes de hipótesis utilizando la distribución Chi-Cuadrado para contrastar la bondad de ajuste alcanzada. A su vez, mediante la diferencia entre los estadísticos de dos modelos sucesivos con distribución asintótica Chi-Cuadrado, se puede contrastar la significancia de cada interacción triple agregada. 
Tanto para 2005 como 2009 el modelo de independencia es rechazado. La hipótesis de asociación homogénea es contundentemente rechazada en 2005; mientras que en 2009 no se rechaza con un nivel del 5\%. Por lo tanto, en 2009, el modelo con todas las interacciones dobles correspondientes presenta un ajuste aceptable. A pesar de ello, en el cuadro A1 se observa que con la incorporación de la interacción triple entre el género, el tabaco y el abuso de alcohol, el modelo muestra una mejora significativa, por lo que se decide ajustarlo,, presentándose sus parámetros estimados en el cuadro A3. Para el año 2005, la incorporación paulatina de interacciones triples va mejorando el ajuste del modelo, aunque no se logra un ajuste aceptable. Recién cuando todas ellas son incorporadas se tiene un buen ajuste del modelo (1). Los parámetros estimados de dicho modelo se presentan en el cuadro A2.

Por lo tanto, para ajustar los modelos, no son necesarias las interacciones de orden cuatro y mucho menos quíntuple (correspondiente al modelo saturado). A su vez los resultados de las estimaciones muestran que con los datos de 2005 existe una asociación conjunta más fuerte entre las diferentes categorías que componen los estilos de vida y el género, respecto al año 2009. Al tener todas las interacciones triples en el modelo del año 2005, la condición de los cocientes de chance condicionados puede ser crucial para la interpretación de los mismos. Por el contrario, para el año 2009 se tendrán interpretaciones más simples aunque sin la mayor riqueza que brindan las interacciones de mayor orden.

Antes de presentar e interpretar los cocientes de chances derivados de los modelos loglineales estimados, se realiza un análisis gráfico de asociación conjunta derivado del MCA.

\section{Análisis conjunto en la elección de estilos de vida}

En las figuras 1 y 2 se presentan las gráficas que resultan del análisis de correspondencia múltiple entre las categorías de interés para los años 2005 y 2009, respectivamente. De las mismas se observan dos dimensiones representadas por un eje vertical y otro horizontal, ambos centrados en el 'cero', los que definen cuatro cuadrantes con tipologías contrapuestas. A su vez, dentro de cada uno de ellos se encuentran las características asociadas por su cercanía respecto a una noción de menor distancia de las mismas. Ambos ejes muestran una clara demarcación entre varones y mujeres, representados en cuadrantes opuestos.

Para el año 2005, en el mismo cuadrante de los varones se encuentra el abuso de bebidas alcohólicas y el nivel de actividad física intenso. Por el contrario en el cuadrante de mujeres se observa una asociación con el no abuso de alcohol y el nivel de actividad física bajo. Por lo tanto, desde una primera inspección gráfica se puede establecer una asociación entre el género masculino y conductas asociadas a la demarcación de la masculinidad. Como es de esperarse, el consumo habitual de frutas $y$ verduras junto con la abstención de tabaco se encuentran contrapuesto al no consumo de frutas y verduras con el hábito de consumo corriente de tabaco. Si se toma el eje vertical se deduce que las mujeres tienen una mayor propensión a no fumar y al consumo habitual de frutas $y$ verduras. Por otra parte, el eje horizontal indicaría lo contrario, motivo por el cual parece haber una menor asociación entre estos hábitos y el género; sin embargo, tomando en cuenta la cercanía de los puntos, se observa que las mujeres tienen una mayor cercanía con estilos de vida relacionados al consumo habitual de frutas y verduras junto a la abstención al tabaco. 
FIGURA 1

ASOCIACIÓN ENTRE EL GÉNERO Y LOS ESTILOS DE VIDA. AÑO 2005

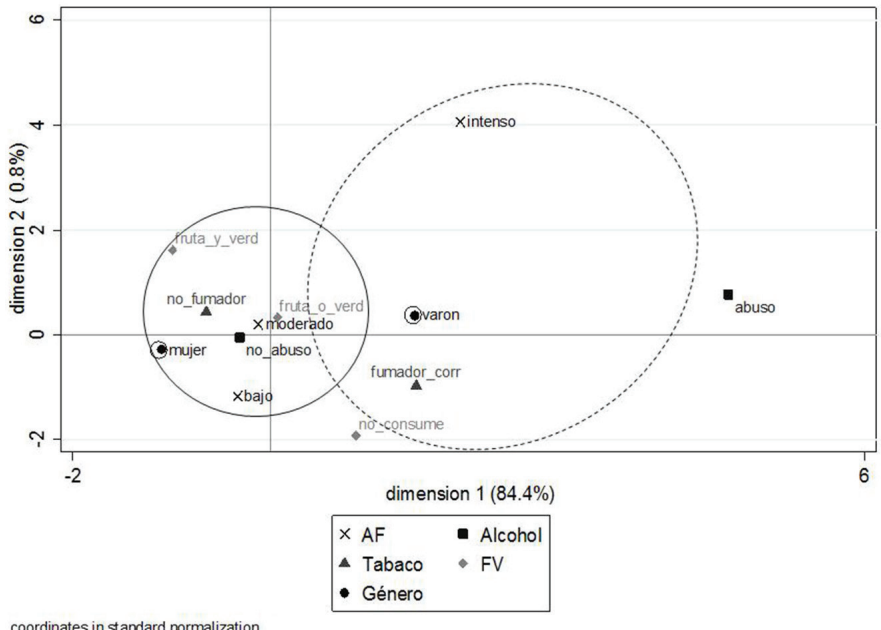

Fuente: Elaboración Propia.

FIGURA 2

ASOCIACIÓN ENTRE EL GÉNERO Y LOS ESTILOS DE VIDA. AÑO 2009

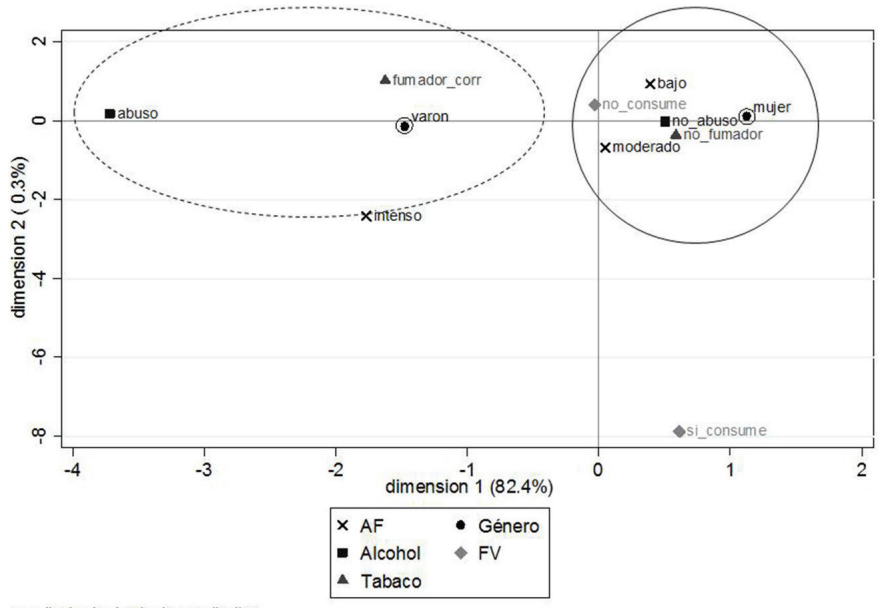

coordinates in standard normalization

Fuente: Elaboración Propia. 
Para el año 2009 también se observa una mayor asociación entre los varones y los hábitos de consumo corriente de tabaco, abuso de alcohol y nivel intenso de actividad física. Para la variable que tipifica el consumo recomendado de frutas y verduras, se observa una menor asociación con el género y el resto de los estilos de vida seleccionados, por lo que no puede establecerse una clara asociación al respecto. Según el eje vertical, a los hombres se les asociaría el no consumir la cantidad recomendada de frutas y verduras, sin embargo ocurre lo contrario según el eje horizontal.

Por lo tanto, del análisis gráfico multivariado se observa una mayor asociación de las mujeres con hábitos saludables. Estos resultados son consistentes con la determinación del sexo fuerte por parte de los varones, con mayor propensión al consumo de alcohol y tabaco, peores hábitos alimenticios junto con una mayor disposición hacia actividades físicas intensas.

\section{Consumo de Tabaco}

En el cuadro 2 se presentan los cocientes de chance condicionados entre el consumo de tabaco y el género derivados de los modelos (1) ajustados para cada año. Los OR muestran que cuando los individuos no abusan de bebidas alcohólicas, la chance de que un hombre sea fumador corriente es aproximadamente un 50\% mayor a la de una mujer lo sea, $y$ tal diferencia es estadísticamente significativa al 1\%. Las estimaciones del año 2005 muestran que esta diferencia es mayor entre personas con menor actividad física. Por su parte, las estimaciones de 2009 muestran que las diferencias de género en el consumo de tabaco solo resultaron significativas entre los individuos que no consumen frutas ni verduras.

Por otra parte, entre aquellos individuos que presentan abuso habitual de bebidas alcohólicas, las diferencias de género no son significativas y adicionalmente los $O R$ muestran una mayor chance de consumo de tabaco en mujeres que en varones.

En 2009 los $O R$ condicionados al nivel de actividad física y al consumo de frutas y verduras no varían dado que las estimaciones mostraron que las interacciones triples con dichas variables no son significativas ni mejoran el ajuste del modelo.

Los resultados para el año 2005 muestran también que las diferencias de género en el consumo de tabaco se apaciguan o desaparecen cuando los individuos tienen prácticas saludables en términos del consumo de alcohol y de la práctica intensa de de actividad física.

Condicionado a los hábitos de consumo de frutas y verduras, para el año 2005 se observa que respecto a los no consumidores, las chances de consumo de tabaco son mayores en hombres cuando consumen frutas o verduras de manera habitual. Esto indicaría que el comportamiento de las mujeres resulta ser más consistente en términos de mantener un estilo de vida más saludable; sin embargo, al observar aquellos que consumen tanto frutas como verduras, los $O R$ se mantienen relativamente constantes en relación a quienes no consumen ni frutas ni verduras, por lo que en este caso las conclusiones son similares a las de 2009; es decir que los hábitos de consumo de frutas y verduras no parecen tener un efecto relevante en la relación de género con el consumo de tabaco. 
CUADRO 2

GÉNERO Y CONSUMO DE TABACO

\begin{tabular}{|c|c|c|c|c|c|c|}
\hline \multirow[b]{3}{*}{$F V$} & \multirow[b]{3}{*}{$A$} & \multirow[b]{3}{*}{$A F$} & \multicolumn{4}{|c|}{ OR (Fumador Corriente, Varones) } \\
\hline & & & \multicolumn{2}{|c|}{2005} & \multicolumn{2}{|c|}{2009} \\
\hline & & & $O R$ & $\mathrm{EE}_{\ln (O R)}$ & $O R$ & $\mathrm{EE}_{\ln (O R)}$ \\
\hline \multirow[t]{6}{*}{ No consumo } & No abusa & Bajo & $1,48^{*}$ & 0,056 & $1,55^{*}$ & 0,036 \\
\hline & & Moderado & $1,45^{*}$ & 0,058 & $1,55^{*}$ & 0,049 \\
\hline & & Intenso & 1,17 & 0,136 & $1,55^{*}$ & 0,075 \\
\hline & Abusa & Bajo & 0,86 & 0,314 & 0,91 & 0,165 \\
\hline & & Moderado & 0,83 & 0,316 & 0,91 & 0,207 \\
\hline & & Intenso & 0,68 & 0,761 & 0,91 & 0,291 \\
\hline \multirow[t]{6}{*}{$\begin{array}{l}\text { Fruta o } \\
\text { verdura }\end{array}$} & No abusa & Bajo & $1,66^{*}$ & 0,057 & 1,55 & 0,187 \\
\hline & & Moderado & $1,62^{*}$ & 0,056 & 1,55 & 0,231 \\
\hline & & Intenso & $1,31^{* *}$ & 0,117 & 1,55 & 0,317 \\
\hline & Abusa & Bajo & 0,96 & 0,378 & 0,91 & 0,834 \\
\hline & & Moderado & 0,93 & 0,324 & 0,91 & 1,160 \\
\hline & & Intenso & 0,76 & 0,678 & 0,91 & 1,189 \\
\hline \multirow[t]{6}{*}{$\begin{array}{l}\text { Fruta y } \\
\text { verdura }\end{array}$} & No abusa & Bajo & $1,47^{*}$ & 0,071 & & \\
\hline & & Moderado & $1,43^{* *}$ & 0,068 & & \\
\hline & & Intenso & 1,16 & 0,126 & & \\
\hline & Abusa & Bajo & 0,85 & 0,458 & & \\
\hline & & Moderado & 0,83 & 0,386 & & \\
\hline & & Intenso & 0,67 & 0,610 & & \\
\hline
\end{tabular}

Nota: * Se rechaza la hipótesis nula Ho: OR=1 a nivel del 1\%, ** a un nivel del 5\%, *** a un nivel del 10\%.

Fuente: Elaboración propia.

\section{Consumo de Alcohol}

Para el caso del abuso de bebidas alcohólicas se encuentran una gran brecha entre varones $y$ mujeres. Entre las personas no fumadoras las diferencias son menores, mostrando que en 2005 los varones tuvieron aproximadamente 8 veces más chance de realizar un consumo excesivo de bebidas alcohólicas que las mujeres. Para 2009 tal brecha se reduce a 6 veces más en hombres que en mujeres. Entre los fumadores corrientes, los $O R$ muestran que en promedio los hombres tienen 13 veces más chances de abuso de alcohol que las mujeres. Estos resultados, junto con los $O R$ condicionados del consumo del tabaco analizados anteriormente, indican que la distancia que existe entre varones y mujeres respecto a un hábito poco saludable es menor cuando los mismos ya incurren en otras prácticas perjudiciales para la salud. 
Condicionado al nivel de actividad física, las diferencias en el consumo de alcohol en 2005 son similares (e iguales en 2009), aunque en el caso de las personas que realizan actividad física moderada las diferencias en las chances de consumo son mayores. De aquí puede concluirse que los hábitos saludables en términos de actividad física tuvieron un mayor peso sobre el cuidado en el consumo de bebidas alcohólicas en mujeres que en varones.

Tomando el consumo de frutas y/o verduras, los $O R$ del abuso de alcohol muestran un comportamiento similar al de tabaco: si bien para la muestra de 2005 la brecha entre hombres y mujeres es mayor cuando las personas son consumidoras de futas o verduras, se mantiene relativamente estable cuando la muestra considera aquellos que consumen tanto frutas como verduras de manera habitual. Por lo tanto, para el caso del abuso de alcohol, los hábitos alimenticios no parecen ser relevantes para explicar las diferencias de género encontradas.

CUADRO 3

GÉNERO Y ABUSO DE BEBIDAS ALCOHÓLICAS

\begin{tabular}{|c|c|c|c|c|c|c|}
\hline \multirow[b]{3}{*}{$F V$} & \multirow[b]{3}{*}{$A$} & \multirow[b]{3}{*}{$A F$} & \multicolumn{4}{|c|}{ OR (Abuso de alcohol, Varones) } \\
\hline & & & \multicolumn{2}{|c|}{2005} & \multicolumn{2}{|c|}{2009} \\
\hline & & & $\mathrm{OR}$ & $\mathrm{EE}_{\ln (\mathrm{OR})}$ & $\mathrm{OR}$ & $\mathrm{EE}_{\ln (\mathrm{OR})}$ \\
\hline \multirow[t]{6}{*}{ No consumo } & Fuma & Bajo & $7,29 *$ & 0,192 & $6,15^{*}$ & 0,113 \\
\hline & & Moderado & $8,55^{*}$ & 0,192 & $6,15^{*}$ & 0,147 \\
\hline & & Intenso & $7,29 *$ & 0,455 & $6,15^{*}$ & 0,204 \\
\hline & No Fuma & Bajo & $12,64^{*}$ & 0,254 & $10,46^{*}$ & 0,126 \\
\hline & & Moderado & $14,83^{*}$ & 0,258 & $10,46^{*}$ & 0,154 \\
\hline & & Intenso & $12,63^{*}$ & 0,625 & $10,46^{*}$ & 0,220 \\
\hline \multirow[t]{6}{*}{ fruta o verdura } & Fuma & Bajo & $9,49^{*}$ & 0,241 & $6,15^{*}$ & 0,627 \\
\hline & & Moderado & $11,14^{*}$ & 0,208 & $6,15^{* *}$ & 0,891 \\
\hline & & Intenso & $9,49 *$ & 0,424 & $6,15^{* *}$ & 0,918 \\
\hline & No Fuma & Bajo & $16,46^{*}$ & 0,297 & $10,46^{*}$ & 0,581 \\
\hline & & Moderado & $19,31^{*}$ & 0,254 & $10,46^{*}$ & 0,778 \\
\hline & & Intenso & $16,45^{*}$ & 0,542 & $10,46^{*}$ & 0,819 \\
\hline \multirow[t]{6}{*}{ fruta $y$ verdura } & Fuma & Bajo & $7,12^{*}$ & 0,326 & & \\
\hline & & Moderado & $8,36^{*}$ & 0,274 & & \\
\hline & & Intenso & $7,12^{*}$ & 0,412 & & \\
\hline & No Fuma & Bajo & $12,4^{*}$ & 0,330 & & \\
\hline & & Moderado & $14,5^{*}$ & 0,280 & & \\
\hline & & Intenso & $12,3^{*}$ & 0,466 & & \\
\hline
\end{tabular}

Nota: * Se rechaza la hipótesis nula Ho: $O R=1$ a nivel del 1\%, ** a un nivel del 5\%, *** a un nivel del 10\%.

Fuente: Elaboración Propia. 


\section{Consumo de Frutas y Verduras}

Si bien las diferencias de género en el consumo de alcohol y tabaco no son sensibles al hábito de consumo de frutas y verduras, sí se observa una mayor chance en las mujeres que en los hombres de consumir habitualmente frutas y/o verduras. De todas maneras, el consumo alimentario como es aquí considerado, no toma en cuenta factores cruciales en relación a la construcción del género, como podría ser la división sexual del trabajo doméstico, $y$ en particular, la preparación de alimentos en el hogar, tarea tradicionalmente atribuida a las mujeres. Como sostiene Borrás Catalá (2007: 154) el consumo, lejos de ser un ámbito igualitario, establece y reproduce la desigualdad de género; sin embargo estas desigualdades no pueden detectarse de los datos disponibles al no contar con información adicional respecto a la distribución de las tareas domésticas del hogar al que pertenece el individuo analizado. Por ello, si los patrones de consumo de frutas y verduras entre hombres y mujeres son similares, ello puede deberse a que la elección del consumo alimentario del hogar está a cargo de las mujeres, $y$ por ello, tanto hombres como mujeres podrían presentar hábitos parecidos, aún cuando existan diferentes motivaciones entre un consumo más o menos saludable. A pesar de esta limitante, los resultados empíricos muestran algunas diferencias dignas de analizar y que aún apoyan la hipótesis planteada respecto a la mayor propensión que tienen las mujeres sobre hábitos alimenticios más saludables.

Para el año 2009, los resultados muestran que la chance de realizar un consumo recomendado de frutas $y$ verduras es un $14 \%(=100 \times 1 / 0,88)$ superior en mujeres que en varones; sin embargo, dicha diferencia no es estadísticamente significativa.

Para la muestra de 2005 se tiene que las diferencias de género son significativas solo cuando no hay abuso de alcohol. Entre quienes no abusan de bebidas alcohólicas se observa que las mujeres tienen aproximadamente un 30\% más chance que los hombres de consumir frutas o verduras y un $62 \%$ más chance cuando se evalúa el consumo tanto de frutas como verduras frente a no consumir ni frutas ni verduras de manera habitual.

Condicionado al consumo de tabaco se revelan menores diferencias de género en el consumo de frutas o verduras cuando son fumadores corrientes (año 2005). Por el contrario, para el consumo tanto de frutas como verduras las diferencias entre varones y mujeres se acentúan cuando son fumadores; sin embargo estas variaciones de los $O R$ son pequeñas, pues no muestran un patrón claro del que puedan extraerse conclusiones más robustas.

Cuando se considera el nivel de actividad física, se observa que entre las personas que realizan actividad física intensa, las diferencias entre varones y mujeres respecto al consumo de frutas y/o verduras son mayores. Así por ejemplo, entre quienes realizan actividad física moderada, las mujeres tienen aproximadamente un $60 \%(100 *((1 / 0,6)-1))$ más chance que los hombres de consumir habitualmente frutas y verduras (vs. no consumo), mientras que entre aquellos que tienen un nivel intenso de actividad física, tal diferencia es del $150 \%(100 *((1 / 0,4)-1))$. Por lo tanto mientras menos sedentarios son los individuos, mayor diferencia se revela en el consumo de frutas $y / o$ verduras entre hombres $y$ mujeres, las que presentan una mayor tendencia a estos hábitos alimenticios más saludables. 


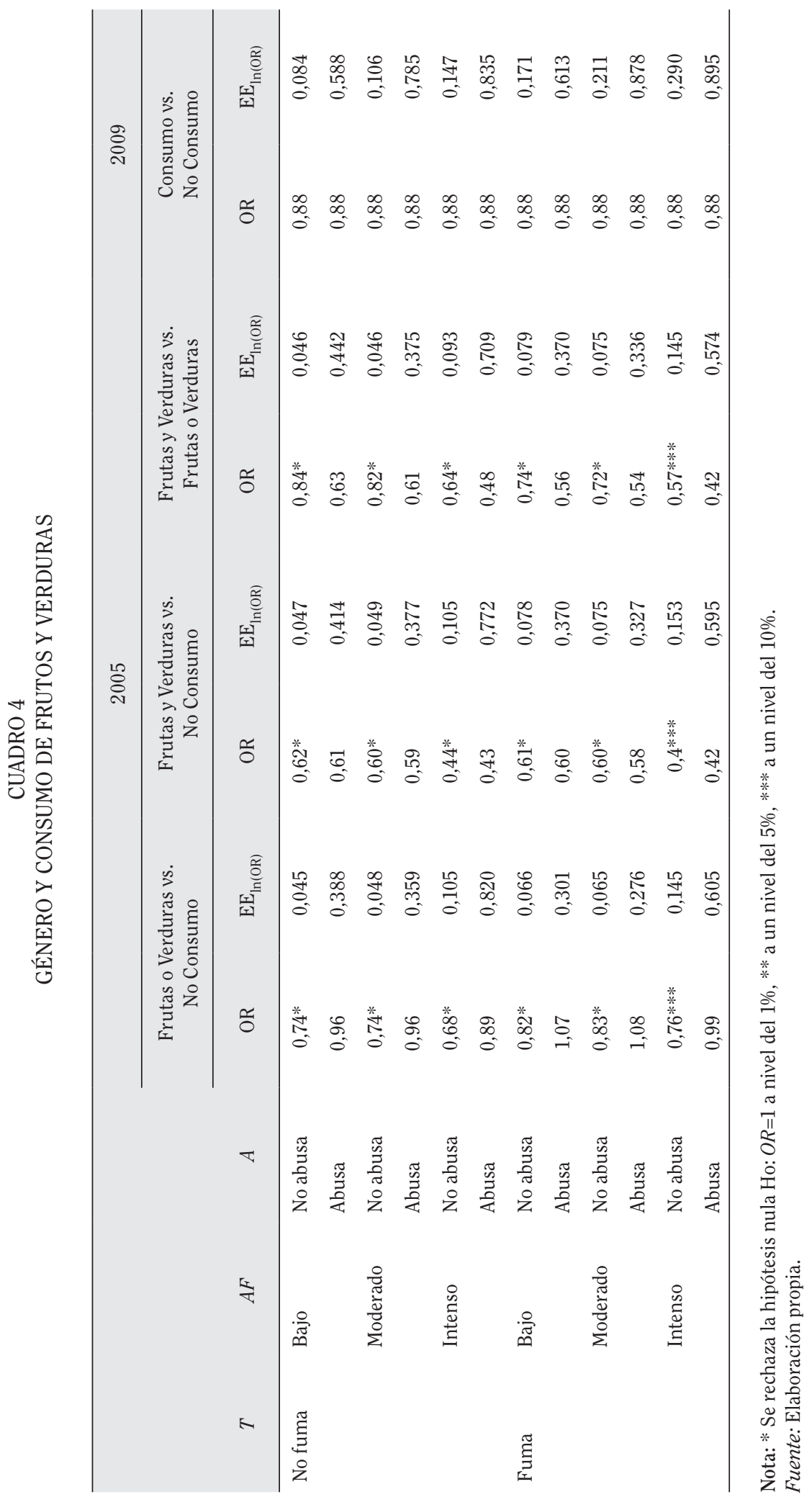




\section{Actividad Física}

Respecto a la actividad física, al comparar el nivel moderado con el bajo los $O R$ son cercanos a 1, por lo que no se evidencian diferencias de género significativas en dichos niveles. Para 2009, se observa que la hipótesis de independencia se rechaza a un nivel del 1\%; sin embargo, las estimaciones muestran que la chance de que los varones realicen mayor actividad física, entre moderado y bajo, es apenas superior en un $8 \%$ a la chance de que las mujeres lo hagan.

Las diferencias de género más relevantes aparecen al comparar el nivel intenso con el moderado y bajo. Se observa que las chances de que un hombre realice actividad intensa llegan hasta triplicar las de las mujeres. Para 2009 los OR son menores, lo cual muestra que la chance de que un hombre practique actividad física intensa es 1,9 veces mayor a la de las mujeres, cuando se toma como base el nivel bajo de actividad física; por otra parte se reduce apenas en un 10\% cuando se toma el nivel moderado como base.

Si bien para 2009 los $O R$ son fijos para cada valor de las variables condicionantes, en el 2005 se observan algunos cambios al igual que se evidenció para las otras categorías del estilo de vida.

Entre los no fumadores, las diferencias entre varones $y$ mujeres son mayores que entre aquellos individuos que tienen el hábito de fumar. A su vez, cuanto mayor es la tendencia a un consumo habitual de frutas y/o verduras, menor es la diferencia de género; es decir que la chance de que las mujeres realicen actividad intensa se acerca más a la de los varones aunque nunca llegue a igualarla.

Por último se puede observar que las diferencias de género respecto al nivel de actividad física son prácticamente independientes de los hábitos de consumo de alcohol.

CUADRO 5

GÉNERO Y ACTIVIDAD FISICA

\begin{tabular}{|c|c|c|c|c|c|c|c|c|}
\hline \multicolumn{3}{|c|}{2005} & \multicolumn{2}{|c|}{ Moderado-Bajo } & \multicolumn{2}{|c|}{ Intenso-Bajo } & \multicolumn{2}{|c|}{ Intenso-Moderado } \\
\hline$T$ & $F V$ & $A$ & OR & $\mathrm{EE}_{\ln (\mathrm{OR})}$ & OR & $\mathrm{EE}_{\ln (\mathrm{OR})}$ & OR & $\mathrm{EE}_{\ln (\mathrm{OR})}$ \\
\hline \multirow[t]{6}{*}{ No fuma } & No consumo & No abusa & 0,99 & 0,048 & $3,25^{*}$ & 0,088 & $3,27 *$ & 0,089 \\
\hline & & Abusa & 1,17 & 0,359 & $3,25^{* * *}$ & 0,668 & 2,78 & 0,670 \\
\hline & Fruta o verdura & No abusa & 1,00 & 0,045 & $3,00^{*}$ & 0,073 & $3,01^{*}$ & 0,073 \\
\hline & & Abusa & 1,17 & 0,389 & $3,00 * * *$ & 0,614 & 2,57 & 0,595 \\
\hline & Fruta $y$ verdura & No abusa & 0,97 & 0,047 & $2,29 *$ & 0,073 & $2,36^{*}$ & 0,073 \\
\hline & & Abusa & 1,14 & 0,430 & $2,28 * *$ & 0,330 & 2,01 & 0,539 \\
\hline \multirow[t]{6}{*}{ Fuma } & No consume & No abusa & 0,97 & 0,065 & $2,57^{*}$ & 0,117 & $2,65^{*}$ & 0,118 \\
\hline & & Abusa & 1,14 & 0,264 & $2,57 * *$ & 0,480 & 2,26 *** & 0,480 \\
\hline & Fruta o verdura & No abusa & 0,97 & 0,067 & $2,38^{*}$ & 0,108 & $2,44^{*}$ & 0,107 \\
\hline & & Abusa & 1,14 & 0,311 & $2,38^{* * *}$ & 0,476 & 2,08 & 0,460 \\
\hline & Fruta $y$ verdura & No abusa & 0,94 & 0,086 & $1,81^{*}$ & 0,125 & $1,91^{*}$ & 0,123 \\
\hline & & Abusa & 1,11 & 0,417 & 1,81 & 0,511 & 1,63 & 0,480 \\
\hline
\end{tabular}




\begin{tabular}{|c|c|c|c|c|c|c|c|c|}
\hline & 2009 & & & & & & & \\
\hline $\mathrm{T}$ & $\mathrm{FV}$ & A & & & & & & \\
\hline \multirow[t]{4}{*}{ No fuma } & No consume & No abusa & $1,08^{*}$ & 0,031 & $1,93^{*}$ & 0,043 & $1,79^{*}$ & 0,046 \\
\hline & & Abusa & $1,08^{*}$ & 0,196 & $1,93^{*}$ & 0,250 & $1,79^{*}$ & 0,264 \\
\hline & Consume & No abusa & $1,08^{*}$ & 0,131 & $1,93^{*}$ & 0,164 & $1,79^{*}$ & 0,175 \\
\hline & & Abusa & $1,08^{*}$ & 0,962 & $1,93^{*}$ & 0,990 & $1,79^{*}$ & 1,115 \\
\hline \multirow[t]{4}{*}{ Fuma } & No consume & No abusa & $1,08^{*}$ & 0,052 & $1,93^{*}$ & 0,071 & $1,79^{*}$ & 0,077 \\
\hline & & Abusa & $1,08^{*}$ & 0,178 & $1,93^{*}$ & 0,222 & $1,79^{*}$ & 0,240 \\
\hline & Consumo & No abusa & $1,08^{*}$ & 0,267 & $1,93^{*}$ & 0,329 & $1,79^{*}$ & 0,351 \\
\hline & & Abusa & $1,08^{*}$ & 1,057 & $1,93^{*}$ & 1,062 & $1,79 *$ & 1,231 \\
\hline
\end{tabular}

Nota: * Se rechaza la hipótesis nula Ho: OR=1 a nivel del 1\%, ** a un nivel del 5\%, *** a un nivel del 10\%.

Fuente: Elaboración propia.

\section{CONCLUSIONES}

En el presente trabajo se expone una investigación empírica destinada a cuantificar las posibles diferencias que existen entre varones y mujeres en comportamientos relacionados a la elección de los estilos de vida para el caso de Argentina. Desde la perspectiva de género y con base en antecedentes empíricos se formula la hipótesis que sostiene la elección de un estilo de vida "más duro" o menos saludable por parte de los hombres. La misma es explicada a partir de su reafirmación como sexo fuerte, tomando una imagen de masculinidad culturalmente afianzada.

A partir de la base de datos de la Encuesta Nacional de Factores de Riesgo de Argentina para los años 2005-2009, se definen cuatro variables conductuales para caracterizar el estilo de vida: consumo de tabaco, abuso de alcohol, consumo habitual de frutas y verduras y el nivel de actividad física realizado. Con esto se lleva a cabo un estudio cuantitativo de asociación empírica, mediante la aplicación de un análisis de correspondencia múltiple y con la estimación de modelos de conteo log-lineales.

En términos generales los resultados hallados son consistentes con la hipótesis planteada. Específicamente se observa que los varones presentan una mayor propensión al consumo de alcohol y tabaco, con peores hábitos alimenticios en frutas y verduras, junto con una mayor disposición hacia actividades físicas intensas, justificado por razones de afianzamiento de la masculinidad en los varones, como también por posturas que hacen hincapié en cuestiones biológicas.

De las estimaciones de los modelos, se encuentra que en 2005 existe una interrelación o asociación conjunta muy estrecha entre las diferentes categorías que componen los estilos de vida y el género, por lo que el modelo ajustado termina incorporando todas las interacciones triples. Por el contrario, en 2009 la asociación conjunta es más débil, y las interacciones dobles son las relevantes en el modelo log-lineal ajustado.

De los diferentes cocientes de chance computados se observa la mayor diferencia entre varones y mujeres en el abuso de bebidas alcohólicas, lo que es esperable dado que el mismo contempla tanto cuestiones fisiológicas como sociales y culturales. Luego en el nivel de actividad física, se observan importantes diferencias de género, en particular cuando se considera el nivel intenso donde los varones tienen más chance de realizarlo que las mujeres. En el tabaco las 
diferencias son muy reveladoras, en especial cuando tanto hombres como mujeres no abusan de bebidas alcohólicas; cuando abusan, ya las diferencias no son significativas, e incluso se revierte el patrón y las mujeres muestran mayores chances que los varones de ser fumadoras corrientes. Para el consumo habitual de frutas y/o verduras se observa una mayor chance en las mujeres que en los hombres, $y$ mientras mayor sea la actividad física que los individuos realizan, mayor diferencia se revela en el consumo de frutas $y / 0$ verduras entre hombres $y$ mujeres.

Al comparar los diferentes cocientes de chance condicionados, en general se observa un comportamiento más consistente en las mujeres, pues si no fuman tienen menos chances de abusar de bebidas alcohólicas respecto a los varones, y si no abusan de bebidas alcohólicas las chances de ser fumadoras corrientes se reduce considerablemente. De lo anterior surge otra conclusión relevante, las prácticas que configuran el estilo de vida son llevadas a cabo de manera más coordinada por las mujeres que por lo hombres, en el sentido que puede caracterizárselo como generalmente positivo o negativo. Es decir, si una mujer lleva a cabo alguna práctica de manera saludable es bastante probable que los demás hábitos también puedan catalogarse como saludables, lo cual no se observa en los hombres. Futuras investigaciones pueden ser llevadas a cabo con el objeto de indagar acerca de las razones que explican esta diferencia.

\section{REFERENCIAS BIBLIOGRÁFICAS}

Agresti, A. 2002. Categorical Data Analysis. $2^{\text {nd }}$ edition Wiley, Ciudad XXX p.

Aktürk D., Gün S. y T. Kamuk. 2007. Multiple Correspondence Analysis Technique Used in Analyzing the Categorical Data in Social Sciences. Journal of Applied Sciences, 7 (4): 585-588.

Backet, K.C. y C. Davison. 1995. Lifecourse and Lifestyle: the Social and Structural Location of Health Behaviours. Social Science \& Medicine. 40 (5): 629-638.

Balia, S. y A. M. Jones. 2008. Mortality, Lifestyle and Socio-Economic Status. Journal of Health Economics. 27: 1-26.

Bates, L.M; Hankivsky, O. y K.W. Springer. 2009. Gender and Health Inequities: A Comment on the Final Report of the WHO Commision on the Social Determinants of Health. Social Science \& Medicine. 69: 1002-1004.

Bauer, T; Göhlmann, S. y M. Sinning. 2006. Gender Differences in Smoking Behavior. Discussion Paper $\mathrm{N}^{\circ} 2259$. IZA.

Borrell, C; García-Calvente, M. y J.V. Martí-Boscà. 2004. La Salud Pública desde la Perspectiva de Género y Clase Social. Gaceta Sanitaria. 18 (1): 2-6.

Borràs Catalá, V. 2007. Las Desigualdades en el Consumo a través del Género. Revista Española de Sociología, 8:139-156.

Bryant, T; Leaver, C. y J. Dunn. 2009. Unmet Healthcare Need, Gender, and Health Inequalities in Canada. Health Policy. 91: 24-32.

Cockerham, W. C. 2000. Health Lifestyle in Rusia. Social Science \& Medicine. 51: 1313-1324.

Cockerham, W.C; Pinote, B.P; Abbott, P. y C. Haerpfer. 2004. Health Lifestyles in Central Asia: the Case of Kazakhstan and Kyrgyzstan. Social Science \& Medicine. 59: 1409-1421.

Cockerham, W.C. 2005. Health Lifestyle Theory and the Convergence of Agency and Structure. Journal of Health and Social Behavior. 46 (1): 51-67.

Cockerham, W.C; Hinote, B.P. y P. Abbott. 2006. Psychological Distress, Gender, and Health Lifestyle in Belarus, Kazakhstan, Russia, and Ukraine. Social Science \& Medicine. 63: 23812394.

Conell, R.W. y J.W. Messerschmidt. 2005. Hegemonic Masculinity. Rethinking the Concept. Gender \& Society. 19 (6): 829-859.

Contoyannis, P. y A.M. Jones. 2004. Socio-economic Status, Health and Lifestyle. Journal of Health Economics. 23: 965-995. 
Courtenay, W.H. 2000. Constructions of Masculinity and Their Influences on Men's Well-being: a Theory of Gender and Health. Social Science \& Medicine. 50: 1385-1401.

Denton, M; Prus, S. y V. Walters. 2004. Gender Differences in Health: a Canadian Study of the Psychosocial, Structural and Behavioural Determinants of Health. Social Science \& Medicine. 58: 2585-2600.

Denton, M. y V. Walters. 1999. Gender Differences in Structural and Behavioral Determinants of Health: an Analysis of the Social Production of Health. Social Science \& Medicine. 48: 12211235.

Frankish, C.J; Milligan, C.D. y C. Reid. 1998. A Review of Relationships Between Active Living and Determinants Of Health. Social Science \& Medicine. 47 (3): 287-301.

García Ruiz, P. 2009. Estilos de Vida y "Reflexividad" en el Estudio del Consumo: Algunas Propuestas. Documento de Trabajo 2009-03. Facultad de Ciencias Económicas y Empresariales Universidad de Zaragoza. $36 \mathrm{p}$.

Herzfeld, T; Huffman, S.K. y M. Rizov. 2011. The Dynamics of the Russian Lifestyle During Transition: Changes in Food, Alcohol and Cigarette Consumption. Working Paper $\mathrm{N}^{\circ} 09019$. Iowa State University, Department of Economics. 40 p.

Hitchman, S.C. y G.T. Fong. 2011. Gender Empowerment and Female-to-male Smoking Prevalence Ratios. Bull World Health Organ. 89: 195-202.

Kiefer, I; Rathmanner, T. y M. Kunze. 2005. Eating and Dieting Differences in Men and Women. Journal of Men's Health \& Gender. 2 (2): 194-201.

Krieger, N. 2003. Genders, Sexes, and Health: What Are the Connections - and Why Does it Matter? International Journal of Epidemiology. 32: 652-657.

Ozden, S Y M. Mendes. 2005. The Usage of Multiple Correspondence Analysis in Rural Migration Analysis. New Medit, 4 (4): 36-41.

Link, B.G. 2008. Epidemiological Sociology and the Social Shaping of Population Health. 49 (4): 367-384.

Lundborg, P. y H. Andersson. 2008. Gender, Risk Perceptions, and Smoking Behavior. Journal of Health Economics. 27: 1299-1311.

Pollard, T.M. y S.B. Hyatt. 1999. Sex, Gender and Health: Integrating Biological and Social Perspectives, p. 1-16. En Pollard, T.M. y S.B. Hyatt, Sex, Gender and Health. Cambridge.

Prus, S.G. 2011. Comparing Social Determinants of Self-rated Health Across the United States and Canada. Social Science \& Medicine. 73:50-59.

Rimal, A.P; Fletcher, S.M. y K.H. McWatters. 2000. Nutrition Considerations in Food Selection. International Food and Agribusiness Management Review. 3: 55-70.

Wilsnack, R.W; Wilsnack, S.C. y I. S. Obot. 2005. Why Study Gender, Alcohol and Culture?, p. 1-23. En I. S. Obot y R. Room. Alcohol, Gender and Drinking Problems. Perspectives from Low and Middle Income Countries.

Zugravu, C.A; Patrascu, D; Prejbeanu, I. y R. Cornelia. 2009. Gender Differences in Nutrition and Lifestyle Attitudes for a Sample of Romanians. The Annals of the University Dunarea de Jos of Galati. 8 p.

World Health Organization. 2007. Gender and Tobacco Control: a Policy Brief. Department of Gender, Women and Health. 12 p. 


\section{ANEXO}

CUADRO A1

BONDAD DE AJUSTE DE LOS MODELOS

\begin{tabular}{|c|c|c|c|c|}
\hline \multirow[b]{2}{*}{ Modelo } & \multicolumn{2}{|c|}{2005} & \multicolumn{2}{|c|}{2009} \\
\hline & $G^{2}$ & g.l. & G2 & g.l \\
\hline Independencia & 8225,85 & 64 & 5431,90 & 41 \\
\hline Asociación homogénea & 106,25 & 45 & 45,11 & 27 \\
\hline$(G, T, A)$ & 86,66 & 44 & 23,86 & 26 \\
\hline$\{(G, T, A) ;(T, A, F V)\}$ & 80,64 & 42 & & \\
\hline$\{(G, T, A) ;(T, A, F V) ;(T, A, A F)\}$ & 77,21 & 40 & & \\
\hline$I^{3}(G, T, A, F V, A F)$ & 20,23 & 20 & & \\
\hline
\end{tabular}

Nota: . $G^{2}=2 \sum_{i j k l m} \log \left(\frac{Y_{i j k l m}}{\widehat{\mu}_{i j k l m}}\right)$. "g.l":grados de libertad.
Fuente: Elaboración propia. 
CUADRO A2

ESTIMACIÓN MODELO LOG-LINEAL AÑO 2005

\begin{tabular}{|c|c|c|c|c|c|}
\hline VAR. & Coef. & VAR. & Coef. & VAR. & Coef. \\
\hline \multirow[t]{2}{*}{ G } & $0,368^{*}$ & $T x F Y V$ & $-0,657^{*}$ & $T x A x I$ & $-0,207^{\text {**** }}$ \\
\hline & $(0,0306)$ & & $(0,0561)$ & & $(0,122)$ \\
\hline \multirow[t]{2}{*}{$T$} & $-0,486^{*}$ & $A \times M$ & $0,278^{*}$ & $A x F O V x M$ & $0,207^{* * *}$ \\
\hline & $(0,0366)$ & & $(0,0925)$ & & $(0,106)$ \\
\hline \multirow[t]{2}{*}{$A$} & $-2,332^{*}$ & $A x I$ & $0,490^{*}$ & $A x F O V x I$ & $0,311^{* *}$ \\
\hline & $(0,0737)$ & & $(0,119)$ & & $(0,137)$ \\
\hline \multirow[t]{2}{*}{$M$} & $-0,166^{*}$ & $A x F O V$ & $-0,329 *$ & $A x F Y V x M$ & 0,180 \\
\hline & $(0,0342)$ & & $(0,0987)$ & & $(0,136)$ \\
\hline \multirow[t]{2}{*}{$I$} & $-1,185^{*}$ & $A x F Y V$ & $-0,757^{*}$ & $A x F Y V x I$ & $0,580^{*}$ \\
\hline & $(0,0489)$ & & $(0,122)$ & & $(0,163)$ \\
\hline \multirow[t]{2}{*}{ FOV } & $-0,0253$ & $M x F O V$ & $0,137 *$ & $G x A x F O V$ & $-0,264^{* * *}$ \\
\hline & $(0,0335)$ & & $(0,0455)$ & & $(0,156)$ \\
\hline \multirow[t]{2}{*}{$F Y V$} & $-0,209 *$ & MxTyV & $0,152^{*}$ & $G x A x F Y V$ & 0,0235 \\
\hline & $(0,0355)$ & & $(0,0485)$ & & $(0,173)$ \\
\hline \multirow[t]{2}{*}{$G x T$} & $-0,394^{*}$ & $I x F O V$ & $0,209^{*}$ & $G \times A x M$ & $-0,160$ \\
\hline & $(0,0454)$ & & $(0,0648)$ & & $(0,144)$ \\
\hline \multirow[t]{2}{*}{$G x A$} & $-2,537^{*}$ & $I X F Y V$ & $0,165^{* *}$ & $G \times A x I$ & 0,000757 \\
\hline & $(0,155)$ & & $(0,0686)$ & & $(0,222)$ \\
\hline \multirow[t]{2}{*}{$G \times M$} & 0,00533 & $G x T x A$ & $0,550^{*}$ & GxFOVxM & $-0,00221$ \\
\hline & $(0,0421)$ & & $(0,137)$ & & $(0,0529)$ \\
\hline \multirow[t]{2}{*}{$G x I$} & $-1,178^{*}$ & GxTxFOV & $-0,110^{* *}$ & $G x F O V x I$ & 0,0781 \\
\hline & $(0,0754)$ & & $(0,0534)$ & & $(0,0917)$ \\
\hline \multirow[t]{2}{*}{ GxFOV } & $0,304^{*}$ & GXTXFYV & 0,0103 & $G x F Y V x M$ & 0,0268 \\
\hline & $(0,0408)$ & & $(0,0592)$ & & $(0,0564)$ \\
\hline \multirow[t]{2}{*}{$G x F Y V$} & $0,478^{*}$ & $G \times T x M$ & 0,0246 & $G x F Y V x I$ & $0,352^{*}$ \\
\hline & $(0,0428)$ & & $(0,0486)$ & & $(0,0937)$ \\
\hline \multirow[t]{2}{*}{$T x A$} & $0,947^{*}$ & GxTxI & $0,234^{*}$ & $T x F O V x M$ & $-0,0277$ \\
\hline & $(0,0869)$ & & $(0,0794)$ & & $(0,0544)$ \\
\hline \multirow[t]{2}{*}{$T x M$} & $0,134^{*}$ & $T x A x F O V$ & 0,117 & TxFOVXI & 0,0219 \\
\hline & $(0,0472)$ & & $(0,0965)$ & & $(0,0871)$ \\
\hline \multirow[t]{2}{*}{$T x I$} & 0,0435 & $T x A x F Y V$ & 0,111 & $T x F Y V x M$ & $-0,00591$ \\
\hline & $(0,0706)$ & & $(0,119)$ & & $(0,0606)$ \\
\hline \multirow[t]{2}{*}{$T x F O V$} & $-0,174^{*}$ & $T x A x M$ & $-0,134$ & $T x F Y V x I$ & $0,180^{* * *}$ \\
\hline & $(0,0489)$ & & $(0,0973)$ & & $(0,0948)$ \\
\hline \multirow[t]{2}{*}{ Constante } & $7,366^{*}$ & & & & \\
\hline & $(0,0242)$ & & & & \\
\hline
\end{tabular}

Nota: Desvío Estándar entre paréntesis. * estadísticamente significativo a un nivel del 1\%, ** a un nivel del 5\%, *** a un nivel del $10 \%$.

Fuente: Elaboración propia. 
CUADRO A3

ESTIMACIÓN MODELO LOG-LINEAL AÑO 2009

\begin{tabular}{|c|c|c|c|}
\hline VAR. & Coef & VAR. & Coef \\
\hline \multirow[t]{2}{*}{ G } & $0,625^{*}$ & $A x T$ & $0,999^{*}$ \\
\hline & $(0,0169)$ & & $(0,0471)$ \\
\hline \multirow[t]{2}{*}{$T$} & $-0,883^{*}$ & $A x F V$ & $-0,207 * * *$ \\
\hline & $(0,0229)$ & & $(0,112)$ \\
\hline \multirow[t]{2}{*}{$A$} & $-2,334^{*}$ & $A \times M$ & $0,178^{*}$ \\
\hline & $(0,0406)$ & & $(0,0501)$ \\
\hline \multirow[t]{2}{*}{$M$} & $-0,597^{*}$ & $A x I$ & $0,608^{*}$ \\
\hline & $(0,0218)$ & & $(0,0554)$ \\
\hline \multirow[t]{2}{*}{$I$} & $-1,214^{*}$ & $T x F V$ & $-0,379^{*}$ \\
\hline & $(0,0274)$ & & $(0,0634)$ \\
\hline \multirow[t]{2}{*}{$F V$} & $-3,044^{*}$ & $T x M$ & 0,0214 \\
\hline & $(0,0513)$ & & $(0,0282)$ \\
\hline \multirow[t]{2}{*}{$G \times A$} & $-2,354^{*}$ & $T x I$ & $0,0962^{*}$ \\
\hline & $(0,0869)$ & & $(0,0373)$ \\
\hline \multirow[t]{2}{*}{$G x T$} & $-0,441^{*}$ & $F V x M$ & $0,159^{*}$ \\
\hline & $(0,0265)$ & & $(0,0562)$ \\
\hline \multirow[t]{2}{*}{$G x F V$} & $0,129^{* *}$ & $F V x I$ & $0,396^{*}$ \\
\hline & $(0,0529)$ & & $(0,0714)$ \\
\hline \multirow[t]{2}{*}{$G \times M$} & $-0,0758^{*}$ & $G x A x T$ & $0,545^{*}$ \\
\hline & $(0,0255)$ & & $(0,118)$ \\
\hline \multirow[t]{2}{*}{$G x I$} & $-0,659 *$ & & \\
\hline & $(0,0347)$ & & \\
\hline \multirow[t]{2}{*}{ Constant } & $8,433 *$ & & \\
\hline & $(0,0140)$ & & \\
\hline
\end{tabular}

Nota: Desvío estándar entre paréntesis * estadísticamente significativo a un nivel del 1\%, ** a un nivel del 5\%, *** a un nivel del $10 \%$.

Fuente: Elaboración propia. 